\title{
Signata
}

Annales des sémiotiques / Annals of Semiotics

4 | 2013

Que peut le métalangage?

\section{Topologie des métalangages dans les textes de sociologie}

Jean-Yves Trépos

revues.org

Édition électronique

URL : http://signata.revues.org/957

DOI : 10.4000/signata. 957

ISSN : 2565-7097

\section{Éditeur}

Presses universitaires de Liège (PULg)

\section{Édition imprimée}

Date de publication : 31 décembre 2013

Pagination : 247-272

ISBN : 978-2-87562-042-2

ISSN : 2032-9806

Référence électronique

Jean-Yves Trépos, "Topologie des métalangages dans les textes de sociologie », Signata [En ligne], 4 | 2013, mis en ligne le 30 septembre 2016, consulté le 31 mars 2017. URL : http:// signata.revues.org/957 ; DOI : 10.4000/signata.957 


\title{
PROPOSITIONS DISCIPLINAIRES
}

\section{Topologie des métalangages dans les textes de sociologie}

\author{
Jean-Yves TrÉPos \\ Université de Lorraine
}

\section{Topologie et topoï : une question d'ontologie}

La probabilité de voir figurer dans les travaux publiés en sciences sociales des langages formalisés (qui référenceraient de la manière la plus évidente ce qu'est un métalangage) me semble faible : tout ce qui formalise les modes d'exposition de nos résultats est en général renvoyé à des lieux spécifiques (légendes des tableaux et graphiques, annexes méthodologiques ${ }^{2}$ ). En conséquence directe, mon propos est bien ici, comme l'annonce le titre, de nature topologique. Au lieu de livrer quelques résultats d'une enquête sur les modes de production d'énoncés de métalangue dans des travaux de sociologie (qu'il faudrait faire), je voudrais partir à la recherche de lieux privilégiés où on pourrait les voir à l'œuvre et y observer les transformations qu'ils rendent possibles - ce que j'appelle une topologie. Cet objectif modeste m'autorise à esquiver un choc frontal avec le débat sur les métalangages (Greimas et Courtès, 1979; Rey-Debove, 1978; Auroux, 1979), qui exigerait une plus forte armature que la mienne, sans pour autant me dispenser de mentionner, à l'occasion, la contribution que pourrait y apporter l'analyse des textes sociologiques. L'un des angles possibles d'entrée dans le problème serait, selon de nombreux spécialistes, la paraphrase (Fuchs, 1995).

1. Je remercie le comité de lecture et les deux lecteurs anonymes pour leurs précieuses suggestions qui m’ont permis de prendre la mesure des implications de certains de mes propos. Je remercie Julie Lefebvre pour la communication de certains de ses textes.

2. Bien sûr, il existe des publications entièrement ou très largement consacrées à l'explicitation de ces formalismes sur l'ensemble d'un texte. Elles ne règlent pas le problème, puisqu'elles ont en quelque sorte fui les lieux où sont exposés les résultats qu'elles permettent de trouver ou de comprendre. 
Qu'est-ce que paraphraser en sociologie? Est-ce, pourrait-on dire immédiatement, travailler à énoncer l'hypertexte scientifique comme une vulgate? Estce rapporter et loger dans le texte des matériaux empiriques? Ces questions ne circonscrivent pas le problème car on peut constater, dans les articles de sciences sociales, une tendance à l'internalisation de ces deux matériaux métalinguistiques : bien souvent (mais dans quelle mesure?), la présence transtextuelle de nombreux travaux de pairs, tout comme l'insertion de comptes rendus d'enquête ne dit presque rien de métalinguistique sur lui et ne fait que mimer sa conformité politique aux canons académiques. Ils fonctionnent comme des topoï (Anscombre, 1995) disponibles pour une stéréotypie de la langue sociologique et on pourrait les considérer comme des leurres métalinguistiques : ici, " méta » veut dire " après »; c'est le texte qui vient en quelque sorte se ranger en serre-file d'une série de textes précédents, en constituant performativement la série. Ces hypertextualités contribuent bien sûr à renforcer l'argumentation sur un plan intrathéorique (à quelques rares exceptions formulant des considérations épistémologiques), mais la redondance qu'apporteraient les discours autres (références, extraits d'entretiens) est en quelque sorte lissée. S'il s'avérait que méta- et inter-textualité ne sont pas automatiquement porteuses d'une force métalinguistique, il y aurait quelque justification à effectuer des repérages précis des conditions dans lesquelles une transtextualité autorise un métalangage dense (où « méta » veut dire "sur»). Une telle réflexion concerne aussi ce qu'A. Compagnon appelle « le travail référentiel ». Elle ne sera pas abordée ici ${ }^{3}$, mais l'interrogation sera l'une des composantes du fil conducteur de mon analyse.

Comment comprendre l'intervention d'un métalangage dans un texte scientifique et spécifiquement dans les sciences sociales? La question a été largement envisagée pour la linguistique et, dans ce numéro, pour la sémiotique, mais je souhaite poser ce problème de manière légèrement décalée, parce qu'un texte de sciences règle toujours par l'écriture des questions politiques. C'est l'interprétation que je propose pour l'existence supposée ci-dessus de deux modalités de métalangages : ce sont des options politiques. Il faudrait dire de "politique ontologique ", parce que cette intervention établit des réseaux d'hypertextualité et d'activités de renfort (sur cette notion : Becker, 1988) qui confèrent un être politique (un mode d'existence dans l'espace de production et de reproduction scientifique) à l'écart métalinguistique. Ontologie? À la différence de l'approche philosophique, on ne considère pas ici une science de l'être en général (l'ontologie), mais une diversité d'imbrications de théories de l'être et de pratiques sociales, qui donnent existence et crédibilité à des domaines différents (des ontologies). Ces ontologies sont d'une certaine manière toujours politiques (comme les politiques sont toujours ontologiques). Annemarie Mol l'exprime ainsi :

3. Voir cependant une analyse du « travail référentiel» en sociologie dans : Trépos, 2011. 
[...] Politique ontologique est un terme composite. Il parle d'ontologie qui dans le langage de la philosophie standard définit ce qui appartient au réel, les conditions de possibilité avec lesquelles nous vivons. Si le terme " ontologie » est combiné avec celui de "politique», alors il suggère que les conditions de possibilité ne sont pas données. Que la réalité ne précède pas les pratiques mondaines par lesquelles nous entrons en interaction avec elle, mais qu'elle est plutôt mise en forme au sein de ces pratiques. Donc le terme politique travaille à souligner ce mode actif, ce processus de mise en forme et à montrer que son être est à la fois ouvert et discuté [...]. (Mol, 1999, p. 74-75 - traduction J.-Y.T.)

Faire ressortir ce qu'il en est d'un monde textuel appelé «intervention d'un métalangage », c'est parler du degré de stabilité de l'agencement du métalinguistique et du linguistique et c'est montrer comment ces ontologies servent d'appui et parfois d'obstacle au déploiement des opérations de connaissance. Ces ontologies sont principalement la mise en forme de tensions entre des processus et des états : s'y intéresser c'est comprendre l'effort que déploient les composantes énonciatives pour s'établir comme telles et ensuite se comporter comme elles se sont établies (ici : selon un format, académique ou d'une revue), alors même que le monde textuel « intervention d'un métalangage » est un monde de passages entre les praxis énonciatives difficiles à contrôler. S'intéresser aux ontologies, c’est s'intéresser au passage lui-même (Moser \& Law, 1999). Cela vaut aussi pour les métalangages. C'est pourquoi la conception d'Annemarie Mol, qui revient à situer toutes les ontologies au même niveau est intéressante pour nous : elle permet de rendre compte de ce qu'il y a d'ontologique dans le fait pour un sociologue d'introduire des éléments métalinguistiques dans le courant de son texte, par une mise en actes qui tend à rester locale. Ce sont ces "agencements de métalangages " ${ }^{4}$, tantôt explicites, tantôt implicites - voire inaperçus - qui nous occupent ici. Et, pour le dire tout net : les agencements métalinguistiques, en tant que mise en dispositif des passages, sont plus intéressants pour nous que les métalangages entièrement constitués comme tels ${ }^{5}$.

Il me paraît dès lors souhaitable de scruter des praxis énonciatives qui auraient un caractère à première vue suffisamment complexe pour permettre d'observer l'agencement. Parmi les praxis énonciatives métalinguistiques que je cible, les plus manifestes me semblent exister dans les textes de manière dispersée. Je vise en particulier les écarts lexicaux : emprunts, interférences et torsions polymorphes des lexiques d'autres disciplines, mais aussi d'autres collectifs de travail, semblent y exister sur un mode aussi improbable que fréquent aux yeux du lecteur moyen. La dimension métalinguistique de ces écarts lexicaux ne tient pas seulement au fait qu'ils font voir la déhiscence interne aux textes, quels qu'ils soient, mais

4. La notion d'agencement (comme celle d'assemblage un peu plus loin) est empruntée à Iain Hardie et David MacKenzie (2007).

5. Bien sûr, il ne s'agit pas de nier l'intérêt, pour les mathématiques, du métalangage intégral que furent au début du $\mathrm{xx}^{\mathrm{e}}$ siècle les différentes tentatives de formalisation. 
aussi et selon moi surtout au fait qu'ils sont des actes d'ontologie énonciative au sens envisagé ci-dessus. Néanmoins, on peut estimer qu'il existe, en sociologie, des lieux textuels privilégiés d'inscription de séquences relativement cohérentes, relativement cohésives et presque systématiques de métalangages ${ }^{6}$. Ce sont : la construction d'objet, les définitions et les élaborations conceptuelles. S’agissant du paratexte, titrage, résumés et mots-clés offrent également des occasions d'observer la mise au travail des métalangages, mais ces itérations sont plutôt discontinues.

Toutefois, les connexions qui indiqueraient au lecteur la dimension de politique ontologique de ces écarts, celles qui diraient : "attention, lecteur, ce qui suit se situe à un niveau méta- ", sont particulièrement codées, au point de n'être point toutes interprétables au premier abord, sinon sur le mode du malentendu. Pourquoi alors s'intéresser de façon systématique aux notes et aux parenthèses? En quoi sont-elles des connecteurs de lecture tout autant que " même ", " pourtant ", "néanmoins ", voire "c'est-à-dire "? Ces deux lieux de l'énonciation, malgré leur caractère assez peu normé, offrent des possibilités intéressantes de saisir la topologie des métalangages en sciences sociales. Ce sont en quelque sorte des passages obligés permettant une distribution des niveaux d'argumentation dans le texte (parenthèses) et dans ses marges (notes) — pour le dire dans des termes aujourd'hui à peu près admis : l'« espace du décroché » (pour reprendre l'expression de S. Boucheron ${ }^{7}$ ) dont je dirais volontiers qu'ils complexifient sans vraiment compliquer, puisqu'on peut aussi décrocher dans la lecture. Elles sont un topos argumentatif situé dans une topique d'écriture. On peut les interroger quant à leur exhibition ou leur recel d'énoncés métalinguistiques, quant au lien ontologique qu'ils établissent entre eux (posant ainsi la question de la continuité et de la discontinuité). Ils permettent aussi d'observer que la distinction langage / métalangage ne se confond pas avec la distinction théorie /métathéorie.

Pour apporter quelques éléments de réflexion systématique sur ces lieux restreints, je retiens une forme de traitement de l'objet qui procède par coups de projecteurs sur des articles récents publiés dans des revues de sociologie et une revue de cultural studies (centrée sur les sciences), mais formant un corpus. Ces revues sont francophones et anglophones, mais mon ambition est contrastive plus que comparative.

Je voudrais établir d'emblée quelques conventions spécifiques d'écriture, contribution métalinguistique explicite à une politique du texte. Les articles faisant l’objet de l'analyse ne sont pas répertoriés en « références bibliographiques », mais

6. C'est l'un des attendus des discussions autour des métalangages : dans quelles conditions peuton y observer de la continuité? Voir : Fontanille, 2003. Je n'ai pas le temps de discuter ici du fait que ce questionnement appellerait un métalangage de niveau $n+1$, puisque l'opposition que je reprends moi aussi à mon compte ici à plusieurs reprises - entre continu et discontinu engage des options de visions du monde. Sur ce point, voir : Trépos, 2011, pp. 161-169.

7. Les potentialités de cette expression sont bien mises en évidence dans : Boucheron, 1999, notamment p. 36. 
dans les notes de bas de page. Les guillemets sont utilisés soit pour citer un extrait d'un autre texte, soit pour pratiquer de manière sténographique une opération métalinguistique : mettre en relief l'autonymie, indiquer un écart de niveau de langue (diastratique), mais jamais pour indiquer une distance critique par rapport au propos (ce que j'appelle des guillemets de dénonciation, ces marqueurs métalinguistiques en quelque sorte honteux). Les écarts diatopiques, c'est-à-dire qui utilisent un terme étranger (e.g. stilus, plus loin) sont en italiques sans guillemets.

\section{La note : un agencement essentiellement intra-théorique}

\subsection{La note palimpseste}

La note est revendiquée par la tradition de l'historiographie critique, depuis que Léopold von Ranke (Grafton, 1998, p. 38-77) et, plus tard, Seignobos et Langlois en ont donné la maxime : « le texte persuade, les notes prouvent ». C'est pourtant une arme à double tranchant, parce qu'elle installe un régime de relations entre texte et infra-texte dont la première caractéristique est de n'être pas (ou rarement) explicité et encore moins justifié : il suffit donc que le lecteur ne puisse avoir prise sur l'une de ces relations et une part de l'argumentation s'affaiblit. À l'inverse, le contrôle de ce régime en fait un redoutable outil de débat ${ }^{8}$. Malgré sa fréquence dans les travaux de sciences sociales - une fréquence que nous mesurerons plus loin - la note ne fait pas l'unanimité : pour certains, les notes sont l'occasion de coquetteries narcissiques, voire de manœuvres dilatoires de l'argumentation (on peut, en note, renvoyer à plus tard l'examen d'un argument, voire prendre une option sur une discussion ou un ouvrage) et beaucoup de nos auteurs contemporains s'en servent comme d'un dépotoir, traitant leurs notes comme l' «égout» de leur texte ${ }^{9}$, sans parler de ceux qui cèdent à une forme d'inflation infra-paginale rappelant fort les jeux de l'Oulipo. La note, sorte de couloir réservé pour autobus argumentatif, permet une écriture à plusieurs vitesses qui présente quelques avantages en termes de gestion du raisonnement et qui autorise des ouvertures stratégiques. $\mathrm{Si}$, comme

8. La polémique Chamboredon - Boudon, dans la Revue française de sociologie en 1984 le montre bien. Accusé par Boudon d'avoir "déposé » une critique basse de l'individualisme méthodologique dans une courte note en bas de page, Chamboredon réplique par un texte où chaque phrase, voire chaque partie de phrase est ponctuée d'une note très longue. Il retrouve ainsi c'est manifestement volontaire si l'on en juge par le style dix-huitiémiste adopté - la pratique des notes au XVIII ${ }^{\text {ème }}$ siècle, « lorsqu'elles servaient à commenter ironiquement le récit raconté dans le corps du texte bien autant qu'à étayer sa véracité »(Grafton, 1998, p. 171).

9. «Comme le crissement de la fraise du dentiste, le sourd murmure de la note en bas de la page de l'historien rassure; l'ennui qu'elle distille, comme la douleur infligée par la fraise, n'est nullement vaine : c'est une partie du prix qu'il faut payer pour toucher les dividendes de la science et de la technologie modernes » (A. Grafton, op.cit., p. 13) L'auteur, en veine de métaphores, traite de la note comme des cabinets : indésirables dans la conversation, sauf lorsqu'ils débordent, ils sont un instrument de la civilisation des mœurs. C'est aussi ce que disait Lacan en avril 1968, dans son célèbre Séminaire de Bordeaux (voir : J. Lacan, Mon Enseignement, Paris, Seuil, 2005, p. 83). 
on l'a suggéré ci-dessus, note est autant un lieu argumentatif (un topos) qu'un lieu physique, on peut se passer de lieux textuels où figureraient des notes, mais sans doute pas de ces moments de la rhétorique scientifique où l'auteur d'un texte a besoin de «changer de vitesse » ou de se situer. En définitive, la note est, toujours selon Grafton, un palimpseste ${ }^{10}$ : superposition de techniques d'époques différentes, rarement harmonisées, rarement explicitées.

G. Genette, qui a effectué une rapide recension des types de notes dans des textes discursifs, en conclut que sa fonction est «de complément, parfois de digression, rarement de commentaire : rien, comme on l'a souvent remarqué, qui ne pourrait sans absurdité se trouver intégré au texte même [...] » (Genette, 1987, pp. 300-301). Il insiste sur l'utilité de la note : «Le principal avantage de la note est en effet de ménager dans le discours des effets locaux de nuance, de sourdine, ou, comme on dit encore en musique, de registre, qui contribuent à réduire sa fameuse, et parfois fâcheuse, linéarité. » (p. 301). Mais surtout, il attire l'attention sur le caractère quasi-textuel de la note, qui «prolonge et ramifie le texte » (Ibid.) et qui, en tant que «détour local» ou «bifurcation momentanée du texte [...] lui appartient presque autant qu'une simple parenthèse" (Ibid.). Cette mise en perspective de la note et de la parenthèse convient assez bien à mon entreprise ici.

\subsection{Le métalangage infrapaginal comme stylos}

Les sociologues font-ils usage des notes? Il me semble qu'il faut d'abord répondre globalement et quantitativement à cette question et prendre la mesure des éventuelles variations culturelles qui affecteraient ou conditionneraient ces usages. J'ai procédé, sur un corpus de quatre revues francophones et anglophones, à l'inventaire des notes (voir le Tableau 1 ci-dessous). Elles ne sont stricto sensu infra-paginales que dans les deux revues françaises. Dans les revues anglophones, on trouve en général une dizaine de notes (repoussées à la fin le plus souvent), quand les textes français en comportent à première vue beaucoup plus. Comme le montre le tableau 1, les deux revues françaises sont plus riches en notes; mais, en volume de texte, les deux revues généralistes leur accordent nettement plus de place, l'une se détachant nettement, même en ratio $(\mathrm{r} 1=\mathrm{n}$. de signes / $\mathrm{n}$. de $\mathrm{p}$. des trois numéros). Ce sont donc des habitudes différentes d'utilisation des notes, moins nombreuses et plus longues ( $\mathrm{r} 2=\mathrm{n}$. de signes / n. de notes) chez les anglophones, qui apparaissent.

Comment les sociologues et les auteurs des autres sciences sociales pratiquentils la note? Elle est nettement moins normalisée chez eux que chez les historiens, mais la diversité des usages ne peut cette fois être référée à des différences culturelles. En d'autres termes, on trouve à peu près les mêmes pratiques dans les

10. Le palimpseste, emblème de toute écriture, pour Roland Barthes : selon lui, le texte est « constitué par un entassement de traces (de formes, de souvenirs, de citations, de censures) » (Barthes R., Le plaisir du texte précédé de Variations sur l'écriture, réédition avec préface de Carlo Ossola, Paris, Le Seuil, 2000, p. 73). 
textes francophones et anglophones. Je négligerai donc sur ce point les bénéfices de l'approche contrastive. Néanmoins, il me paraît possible de catégoriser ces pratiques et ainsi d'y situer les usages des métalangages. On pourrait, pour recourir à une typification sommaire, rencontrer deux grands ensembles ${ }^{11}$.

Le premier correspond assez bien à la pratique des historiens : il s'agit d'un texte auxiliaire. Il soutient la théorie que tente d'établir le texte principal, il l'allège, lui permet de respirer parce qu'il autorise la lecture en deux temps (on peut se servir de la note ultérieurement). Il porte les pièces justificatives de l'énoncé principal : la note exhibe les sources, voire certaines données plus complètes que dans le texte (pourcentages, extraits d'entretiens et autres matérialisations du travail accompli), elle comporte des citations développant un propos qui a été utilisé dans l'argumentation du texte principal, des références bibliographiques codées ou entières ${ }^{12}$. Elle peut aussi avoir un caractère plus directement argumentatif : elle apporte des précisions de vocabulaire (définitions, distinctions, etc.), elle discute une thèse concurrente. La nature supplétive de ces notes en fait généralement un texte complètement discontinu : ce n'est qu'en de rares exceptions que l'on peut voir le texte de soutien établir un lien anaphorique entre des pages. On pourra donc trouver dans ce type de note une première occurrence de métalangage qu'il est d'ores et déjà possible de désigner sommairement : définitions, précisions méthodologiques, voire indications biographiques (l'effet «comment j'ai écrit certains de mes livres ») servent à dire quelque chose sur le texte principal; quelque chose qui n'y a pas trouvé sa place, mais qui le soutient en restant en quelque sorte à l'intérieur de l'espace que dessine et peut-être circonscrit ce texte principal, inévitable polyphonie qui se voudrait pourtant plain-chant.

Le second type de note a plutôt un statut métathéorique : il s'agit d'un texte parallèle ou additionnel. L'auteur tient un deuxième discours, aussi fragmentaire et décousu que dans le type précédent, mais qui prend ancrage dans le propos pour s'en éloigner ponctuellement, ce qui donne par différence un statut épistémologique aux deux discours. Cet infra-texte parallèle peut construire des alternatives, c'està-dire : mettre, en regard d'une façon de problématiser qui est le propos actuel, au moins une autre que l'on ne peut ou ne veut retenir, mais qui aurait quelque raison d'être en d'autres circonstances et vers laquelle (lesquelles) on dirige le lecteur avec

11. Cette catégorisation reste sommaire, au regard du travail qui pourrait être entrepris à partir des travaux de Julie Lefebvre, qui prennent en compte les marques de cadrage d'un acte d'énonciation représenté (Lefebvre, 2004). Par exemple, il n’y a ici que peu ou pas de remarques sur les circonstants.

12. Les nouvelles normes d'écriture de Sociologie du travail, liées à un changement d'éditeur, spécifient qu'il ne saurait être question de faire figurer des références en note : "Notes de bas de pages. Elles ne doivent comporter aucune référence bibliographique, ni appel de référence bibliographique" (Sociologie du travail, 41-2, 1999, p. 232). Aucune justification n'est donnée en particulier pour cette norme. Il est vraisemblable que le refus de Sociologie du travail d'inclure des références en bas de page a fait baisser le nombre de notes, rapprochant ainsi la revue du modèle anglophone. 
bienveillance $^{13}$. Mais il me semble que cette catégorie de notes est le plus fréquemment l'occasion d'un propos d'ouverture vers des approfondissements visés par le scripteur lui-même : chacun l'aura remarqué, les auteurs invoquent souvent en note le manque de place ( dans le cadre limité de cet article, on ne saurait...»), pour ne pas poursuivre plus avant un propos (on esquisse des analyses en cours ou à venir ou on renvoie à des textes où l'on a traité cette question), pour suggérer des connexions avec des domaines voisins (qui sont autant de " pistes à explorer») ou encore pour justifier une expression synthétique qui met fin à l'exploration de ces fameuses pistes (soit l'énoncé fictif : « Il faudrait pouvoir citer intégralement les propos d'Untel qui ont véritablement inauguré les travaux de sociologie sur la note en bas de page »).

Certaines notes mêlent texte auxiliaire et texte parallèle. Par ailleurs, la grande majorité des textes n'est pas constante dans la pratique infra-paginale. Essayons néanmoins cette grille d'analyse sur un article de sociologie paru dans une revue française ${ }^{14}$. L'article a recours à 34 notes en bas de page, ce qui le place parmi les gros utilisateurs de ce dispositif textuel. Elles sont presque toutes du premier type (auxiliaire ou intra-théorique), mais elles appuient le texte principal en utilisant plusieurs agencements argumentatifs.

- Un agencement d'empiricité. Le plus grand nombre de notes apporte des arguments tirés de l'enquête, soit en précisant des conditions de déroulement sur un mode plutôt narratif (avec parfois des extraits d'entretiens), notamment en donnant des informations circonstanciées sur les entreprises concernées (n.1, $15,17,19,21,22,24,25,29)$, soit en livrant certains résultats de l'enquête ellemême (n. 18) ou d'une enquête antérieure congruente (n. 20) ${ }^{15}$.

13. Voir par exemple, dans un article de Michel Callon et Vololona Rabeharisoa, la note suivante : « Notre objectif n'est pas de discuter la thèse principale de Peneff, celle de la stabilité du travail du chirurgien. Signalons cependant pour un point de vue différent qui met en évidence la variabilité des rôles du chirurgien dans le cas des interventions à cœur ouvert, variabilité qui est en grande partie liée à l'évolution des techniques, le papier de G. Lawrence [...] » (Callon M. et Rabeharisoa V., « De la sociologie du travail appliquée à l'opération chirurgicale : ou comment faire disparaître la personne du patient ", Sociologie du travail, 41-2, 1999, p. 145, note 3). Cette autre modalité pour l'argumentation est surtout l'affirmation d'une autre option possible face à une thèse non discutée de l'auteur avec lequel Callon et Rabeharisoa débattent par ailleurs.

14. Eustache D., « Politique salariale, régulation et échange social », Revue française de sociologie, 422, 2001, p. 295-326. Le choix de cet article est le résultat d'un tirage à l'aveugle dans des numéros de grandes revues françaises. Il n'implique aucun jugement sur le caractère emblématique de l'article, qui est cependant assez représentatif de l'aspect multiforme ou hybride de l'appareil infrapaginal généralement utilisé.

15. La note 17 illustre un propos résumant («Il [le salarié] peut critiquer la façon dont le hiérarchique encadre et organise son travail ») par une anecdote tirée de l'enquête, qui est rapportée sur un mode narratif ( À̀ l'occasion d'une de nos enquêtes, les informaticiens d'une grande entreprise chimique nous avaient fait part de problèmes d'organisation [...] Au retour de son stage, l'informaticien eut un second entretien d'appréciation avec son supérieur au cours duquel il parvint à le convaincre de suivre la même formation. » (Eustache, op. cit., p. 311). 
- Un agencement hypertextuel. Les notes contiennent un fort appareil référentiel de soutien (notes : $3,4,5,6,7,8,12,27,30,31,33$ ), qui utilise la citation, comme dans la note 3 : «Ainsi que le dit M. Mauss (1925), le producteuréchangiste sent [...] qu'il échange plus qu'un produit [...] »; mais aussi la traduction au sens de Callon (note 8 : « Nous paraphrasons ou plus exactement transposons à notre analyse de la régulation une remarque de F. Furet (1978). L'auteur évoque à ce propos [...]" - suivent trois citations de Furet, à leur tour renforcées par une référence à Boudon pour sa théorie des effets pervers). Participent aussi à ce dispositif référentiel de soutien divers états de vulgates $^{16}$ : note 5 (le don selon Mauss), note 6 (la rationalité limitée selon March et Simon), note 30 (le développement de formes d'autonomie dans le travail selon Crozier). Elles discutent également des théories antérieures. Ainsi, la note 33 dit : « Ce constat tend à affaiblir l'hypothèse d'une professionnalisation des marchés internes d'entreprise en France, qui serait fondée sur le développement de la formation et de la transversalité non seulement interne, mais aussi externe, des compétences (Géhin et Méhaut, 1993)».

- Un agencement de généralisation. Parfois, l'argumentation principale est soutenue par des montées en généralité à partir de l'enquête elle-même : c'est le cas pour la note 14 ( D'où la place importante donnée dans les accords aux mesures transitoires [...]») et pour la note 23, qui procède par mise en équivalence : "Ainsi, la direction générale d'une multinationale nordaméricaine de l'informatique a décidé de redresser [...]»). La fonction de généralisation peut aussi être assurée par le recours à des résultats plus généraux et considérés comme établis (notes 11, 16, 26, 27, 28) : par exemple, la longue note 27 (1232 signes), qui vient soutenir l'idée que les entreprises procèdent par compromis successifs autour d'outils de gestion, permet à l'auteur de montrer la complexité de ce point (en gros : un va-et-vient entre contraintes d'outils et contraintes d'acteurs), en s'appuyant sur deux travaux de référence.

- Un agencement définitionnel ${ }^{17}$. Certaines notes définissent des termes : la note 9 commence par une définition («Par le terme négociation, nous entendons non seulement le processus par lequel la direction et les salariés [...]»), puis elle se poursuit par l'introduction d'une notion non définie, mais que

16. Le sens usuel de vulgate (qui renvoie à un affadissement ou une édulcoration) n'est pas le seul qu'il faille envisager : la vulgate est aussi la réélaboration performative d'un texte X par un texte $\mathrm{Y}$ (la citation par Y sacralise $\mathrm{X}$ au nom de la sacralité de X); ou encore : la mention d'un texte comme s'il n'avait qu'une seule interprétation possible; ou : sa citation sèche (ex : "Mauss, 1968 ») comme si tout l'ouvrage mobilisé disait précisément ce que le texte lui fait dire en ce point de l'analyse. Voir: Trépos, op. cit., pp. 33-38.

17. Le caractère métalinguistique de la définition est plus difficile à établir qu'on ne l'imagine, parce qu'on appelle parfois définition ce qui ne l'est guère (Rey, 1979). Sur la définition en sociologie, voir Trépos, 2011. 
l'auteur considère sans doute comme pouvant être déduite de la définition ( « $\AA$ cet égard, le refus des salariés de s'engager dans une action, leur simple retrait, témoignent d'une quasi-négociation [...]»); elle se poursuit par une argumentation de soutien sous la forme d'un renvoi à la suite ("voir infra l'analyse des réactions des salariés [...]») et sous la forme d'une référence à un ouvrage complémentaire («voir aussi C. Gavini (1998) en ce qui concerne [...]»). Il arrive aussi qu'elles revendiquent un usage rigoureux d'une expression stéréotypée (comme je viens de le faire moi-même à propos des vulgates). Pour que le lecteur comprenne l'expression : "[...] des politiques de "hauts salaires" ", l'auteur écrit en note 10 : "C'est-à-dire des politiques consistant à rémunérer systématiquement, pour des qualifications comparables, les salariés de l'entreprise à des niveaux sensiblement supérieurs à ceux du marché externe ».

- L'article comporte aussi deux notes qui ont intégralement un caractère d'agencement métathéorique : des approfondissements possibles sont désignés, en note 13 : "Une analyse fine de cas d'entreprise montrerait [...]»; des hypothèses à vérifier sont esquissées en note $34:$ : [...] Dans cette hypothèse, l'entreprise ne chercherait pas seulement à [...] On ne peut exclure qu'elles cherchent aussi à mettre en cause [...]»). Pour autant, le recours au métalangage n'est pas homogène ici. La note 13 situe l'" analyse fine de cas " comme un approfondissement qui utilise pleinement les ressources exposées dans l'article mais sur un autre " cas " : à première vue métathéorique (l'analyse fine est distinguée de l'analyse tout court), elle n'a pas pour autant une fonction de métalangage dense (il est sous-entendu que l'article lui-même traite d'un cas et qu'il est porteur potentiel de cette analyse fine). Pour cette note, la paraphrase, sans être tout à fait le «leurre métalinguistique » évoqué au début de cet article, ne nous dit rien par exemple sur le rapport entre « analyse » et « analyse fine». Mais la note 34 combine métathéorie et métalangage : le texte fait état - en utilisant «voire» - d'une possibilité qui excède sa capacité démonstrative actuelle («[...] les entreprises transforment le fonctionnement de leurs marchés internes, voire cherchent à modifier en profondeur leur modèle de relation d'emploi (34)») et la note, en utilisant le conditionnel ( "chercherait») et la réserve ( quel statut épistémologique (une " hypothèse » concernant une "tendance ») aurait cette éventualité. Il me semble que l'on voit bien sur ce cas comment le discours sociologique peut utiliser la note pour déployer un métalangage maîtrisé et, disons-le en jouant sur la polysémie : discret.

On peut d'ailleurs étendre cette remarque aux différents emplois dévolus aux métalangages (définitions et hypertextualité) dans les notes auxiliaires examinées ci-dessus. Pourtant, cette discrétion (comme discontinuité et/ou comme politesse) est trop récurrente dans les textes de sciences sociales pour n'être pas une forme de l'académisme. Ce que j'appellerais volontiers un stylos, en reprenant une 
distinction proposée par Jean Gayon entre stylos et stilus (Gayon, 1998) ${ }^{18}$. Les consignes, les conseils, les prototypes, les examplars (au sens de Kuhn, 1970) et autres standards académiques ont pour effet de délimiter un cadre pour l'écriture, c'est-à-dire de procéder à son encadrement. Il en va de la survie de la pratique ici : de sciences sociales - que de pouvoir être instituée (et donc conservatrice, si l'on en croit les durkheimiens). En ce sens, le présupposé d'une forte dissémination des conventions tacites au sein de ce qu'il est convenu d'appeler la communauté scientifique semble dispenser de recourir systématiquement au métalangage pour caractériser des choix théoriques. Considérons par exemple l'hypertextualité : l'acte énonciatif qui insère dans un texte un prélèvement ou une traduction (au sens de Callon) d'un autre texte pourrait justifier qu'une note indique le contexte dans lequel s'est effectué le prélèvement et donc expliciter, par effet de métalangage, le degré de torsion effectué sur le texte prélevé. Je prie mon lecteur de croire que, selon mon expérience, rien ne va dans ce sens. Je rappelle qu'à mes yeux il s'agit là d'ontologies et je propose donc d'appeler stylos cette dimension que l'on pourrait dire apollinienne du style scientifique. Serait, à l'opposé, stilus tout ce qui singularise, éventuellement déborde et subvertit, ainsi, plutôt dionysiaque. Cette opposition devrait sans doute être dialectisée pour chercher à saisir à quel moment et jusqu'à quel point l'apparition d'une dose de singularité dans une pratique gouvernée par la conformité modifie les formes d'affirmation de soi scripturales, sur un curseur allant de l'obsequium à la tentation prophétique, en passant par diverses nuances de transgressions mesurées. Ma thèse sur ce point serait donc de concevoir une forme dominante d'utilisation infrapaginale des métalangages en sociologie comme présence discrète et souvent non assumée, bien qu'elle puisse être parfois un écart stratégique jouant sur le malentendu, au risque de l'allodoxia, cette méprise dont parle Platon dans le Théétète (189b-190e).

\subsection{Le métalangage comme stilus}

Pourrait-on donc se servir des métalangages comme d'un test pour évaluer certains écarts d'écriture en sciences sociales? Sans doute pas comme instrument de mesure unique, mais peut-être comme indicateur suggestif. Pour esquisser cette entreprise, je retiendrai une pratique d'écriture, celle d'un sociologue qui utilise la littérature comme métalangage, jusqu'aux aux limites mêmes de l'utilisation de la littérature comme langage sociologique. C'est cette ambiguité concernant la frontière des deux disciplines qui est intéressante ici. Qu'il s'agisse là d'un stilus ne fera pas de doute si l'on observe la polémique déjà ancienne entre ce sociologue connu et une revue de sociologie, polémique qui porte sur ce qu'est une écriture acceptable et qui montre bien le rôle que peuvent y jouer les métalangages.

18. La réutilisation que j'en fais ici est tout à fait conforme à l'esprit de la démarche de Gayon dans son article. 
La pratique des notes infrapaginales chez Michel Verret ${ }^{19}$, relève d'un style d'écriture scientifique cohérent et réfléchi, qui confère notamment un statut spécifique (on pourrait le dire d'un mot : provocateur) à la littérature et à la philosophie comme métalangages ${ }^{20}$. À première vue, cette pratique est claire : le texte établit les arguments fondamentaux et, en note, les citations littéraires décalent le texte, le rendent aérien, selon une technique globalement métaphorique. Le texte sociologique lui-même a des accents plutôt littéraires : "Que cette richesse paradoxale ne nous cache pourtant pas son prix : d'incertitude, d'angoisse, de tension, d'échec. Ni ses bornes d'égoïsme ${ }^{a}$ ». Mais la note a l'est explicitement : " "Peu productifs, facilement vulnérables, ils ne peuvent se payer la moindre grandeur d'âme ni tenir le coup qu'au prix d'un égoïsme extrême, même dans les petites choses [...]". B. Brecht, Journal de travail 1938-1955, Paris, L’Arche, 1973, p. $147 »^{21}$. Ici, le métalangage littéraire est en note pour dire en gros la même chose que le texte principal sociologique. L'argumentation progresse en parallèle sur deux fronts.

Pourtant, un examen plus attentif nous montre que le métalangage littéraire ou philosophique peut viser aussi à intégrer pleinement la démonstration au lieu de seulement la décaler. Dans ce cas, le littéraire ou le philosophique perdent leur fonctionnement métaphorique : ils quittent parfois la note pour entrer par effraction dans la lingua sociologica et ils participent à l'établissement syntagmatique du sens. À propos du goût du commun chez les ouvriers, le texte de Michel Verret (paru à peu près en même temps que La Distinction de Bourdieu) dit : "De là ces données communes du logement ouvrier : le peu d'attention, sinon le rire ("le risible, c'est l'impratique", disait Brecht) ${ }^{\mathrm{b}}$, portés “aux objets de parade et d'inutilité", qui composaient "le décor hiérarchique"c des classes dominantes. La présence bien en vue de l'objet simple, "pas du tout plein de son importance, plein par contre de son utilité"d $[\ldots]$ ». Les notes b et $c$, donnent sobrement la référence : "Brecht, op. cit., p. $550 »$; « Le Corbusier, op. cit.». La note d, qui explicite le passage entre guillemets est elle plutôt métaphorique : "Comme le dit Ponge de la lessiveuse : Pièces, éd. cit., p. $75 »^{22}$. Il pourrait y avoir doute sur la nature sociologique des formules entre guillemets, puisque c'est le langage naturel non technique qui est employé, mais les notes b et c se chargent de rappeler leur statut de métalangage. On notera, par provision, une utilisation de la parenthèse comme enveloppe pour un métalangage.

19. Les appels de notes figurant dans les textes de M. Verret ont été transformées en appels alphabétique pour éviter toute confusions avec mes propres notes.

20. L'auteur, agrégé de philosophie, nourri de Brecht et de Musil, professeur de sociologie, fut président de l'Association Française des Sociologues. Il a par ailleurs produit une œuvre poétique.

21. Verret M., La culture ouvrière, St-Sébastien, ACL Editions, 1988, p. 42 et 52.

22. Verret M., L'espace ouvrier, Paris, A. Colin, 1979, p. 142 et 221. 
La provocation peut aller plus loin, chez le même auteur en inversant les places du littéraire et du sociologique. En 1984, M. Verret propose à la Revue française de sociologie deux comptes-rendus de lecture d'un ouvrage de P. Hughes sur le tissu. Il écrit : "Lui qui se donne aux sens muets : la peau et l'œile »; puis directement en dessous, avec saut de ligne : «Lui qui se donne d'un coup ${ }^{\mathrm{f}}$, sans avoir à dérouler la chaîne parlée [...]»; et, un peu plus loin : «- une structure continue, indéfinie, pluridirectionnelle ; plane, bien que parfois, velours, elle se donne une profondeur ${ }^{\text {h }}$ [...]». Les notes, lapidairement hypertextuelles, désarçonnent celui qui attendrait quelques précisions sur un ouvrage qu'il pourrait se proposer de lire : e «id. p. 7 »; f «id. p. $13 » ;$ g «id. p. $46-50 » ;$ h «id. p. 25, p. $219 »$. Pourtant, l'ambition de l'auteur n'est pas seulement de provoquer, mais de créer une véritable rubrique pour un autre type de comptes-rendus, qu'il décide d'appeler «Parcours ». La rédaction de la revue ne l'entend pas ainsi :

Le premier mouvement de notre Comité de rédaction avait été de rejeter la publication des deux textes qui suivent, parce qu'il les jugeait trop éloignés des formes admises dans les revues scientifiques en matière de comptes-rendus critiques et trop peu informatifs. L'auteur a fait appel de ce refus [...]

La protestation de M. Verret mobilise diverses ressources (y compris celle d'une fonction de représentation de la profession qu'il occupa jadis), mais surtout l'auteur refuse de se soumettre à l'idée qu'il puisse y avoir des «formes admises dans les revues scientifiques ", interpellant les rédacteurs sur un mode rhétorique :

Et, pour parler précis, un texte dans le genre et l'écriture de ces Parcours a-t-il sa place dans la revue ? ${ }^{23}$.

Cette écriture elliptique et qui brouille les frontières marque bien comment un stilus peut résulter du parti pris de jouer avec le métalangage, sans toutefois aller jusqu'à thématiser l'opération, ce qui en atténue l'écho. À ma connaissance, cette entreprise d'écriture est demeurée presque sans postérité.

On ne peut sans doute pas tirer de ces deux exemples (Eustache et Verret) des enseignements qui auraient quelque chance de valoir pour l'ensemble des écrits sociologiques. Mais ces deux études de cas auront permis de passer en revue quelques uns des agencements de métalangages dans les notes des textes de sociologie : agencements définitionnels, hypertextuels, méthodologiques, épistémologiques. Ces pratiques sont très rarement thématisées et fonctionnent sur le mode de la coexistence avec les éléments d'argumentation directe, par lesquels l'auteur établit sa démonstration. Les incursions de métalangages ont ainsi essentiellement un rôle intra-théorique et ne contribuent par exemple presque jamais à faire entrer explicitement aux marges du texte tout ce qui l'a fait texte : le contexte, les prétextes (les textes précédents qui fournissent leur caution).

23. Revue française de sociologie, 25-4, 1984, p. 703 à 710 . 


\section{Les agencements parenthétiques}

Par une opération en quelque sorte parallèle à la précédente, je vais maintenant m'intéresser aux écarts qu'introduisent les parenthèses. Leur rapport avec les notes en bas de page n'est pas toujours clair (je précise au passage que je ne ferai pas de traitement spécifique ici des parenthèses figurant en notes). Les parenthèses - sil'on exclut celles, conventionnées, qui servent à énoncer les renvois bibliographiques sont souvent un moyen économique d'insérer une précision sans perturber exagérément la lecture ${ }^{24}$. Peut-on parodier l'Argumentation dans la Langue et considérer que la parenthèse fixe le degré de généralité de la phrase dont elle semble n'être que l'excroissance ou la dégénérescence? On peut en tout cas s'attendre à ce qu'elle soit un lieu privilégié d'inscription des métalangages. Par rapport aux notes, il s'agirait donc d'une topique différente pour une topologie semblable.

\subsection{Parenthèses syntagmatiques et paradigmatiques, ici et ailleurs}

Dans son « plaidoyer » pour les parenthèses, contre l'accusation de ventripotence, Sabine Boucheron propose une interprétation du décrochement parenthétique comme l'établissement d'un espace à part entière, qui permet à un discours et à un sujet de singulariser. Elle note que c'est

[...] dans cette baisse de "tension syntaxique » que l'on trouve notamment de longues énumérations, c'est-à-dire des paradigmes amplement rabattus et allègrement déployés - grâce aux parenthèses - sur l'axe syntagmatique (et qui constituent souvent des listes d'hyperonymes d'un mot appartenant à la phrase insérante) (Boucheron, 1997, p. 11).

Sur la base de ce premier constat, on peut établir un un premier plan d'analyse, pour distinguer plusieurs catégories d'agencements paradigmatiques et d'agencements syntagmatiques des parenthèses ${ }^{25}$. Dans les agencements paradigmatiques,

24. La parenthèse ne plaît guère. Armand Hadria et Pierre Gaussot — qui signalent fort opportunément que le mot vient du grec parenthesis, i.e. action de placer à côté - estiment que le plus souvent « la parenthèse, remarque incidente ou digression, nuit à la clarté de l'exposé et constitue une surcharge qu'il faut bannir parce qu'elle interrompt la construction syntaxique et le déroulement clair de la pensée » (Hadria A. \& Gaussot P., "Du bon usage des parenthèses », Défense de la langue française, $\mathrm{n}^{\circ} 225,2007$, p. 36). Si l'usage des parenthèses heurte les amoureux d'une langue sans surcharge, il a aussi conduit Łukaczsiewicz à proposer en 1920 une écriture logique plus rigoureuse quoique moins lisible (la notation préfixée, dite " notation polonaise ») et d'autres à prendre un parti symétrique (la notation polonaise inverse). Voir : Dupas, 2008.

25. Je reprends ici, sans y embarquer la controverse nourrie par Chomsky et peut-être dépassée (Vandendorpe, 1990), la distinction saussurienne parce qu'elle permet une navigation simple dans ces eaux. Il eût peut-être été préférable d'opter pour la distinction mise en œuvre par Jean Cavaillès, à propos d'une possible théorie de la science, entre le paradigmatique et le thématique. Est paradigme le "moment de la variable», "l'actualisation » c'est-à-dire " [...] un rapport qui en tant que tel ne s'affirme que dans la singularité de réalisation de l'enchaînement, mais ne réclame cette singularité que quelconque, donc, tout en la posant, la supprime et révèle par là un principe interne de variation " (Cavaillès, 1960, p. 29 et p. 27) - les caractères gras 
le contenu de la parenthèse est substituable à ce qui le précède : soit il s'agit de substituer un terme à un autre en restant au même niveau argumentatif (appelonsla : parenthèse homologique), soit il s'agit de substituer un terme équivalent non développé à un terme développé dans l'énoncé (ce qu'on pourrait appeler la parenthèse heuristique). On peut, d'un autre côté, appeler syntagmatiques : soit des parenthèses illustratives, qui insèrent un matériau expérientiel ${ }^{26}$ ou issu d'études empiriques soit des parenthèses incidentes qui intègrent à l'énoncé un complément de raisonnement, qui n'est pas strictement nécessaire à sa validité ${ }^{27}$.

Peut-on, dans ces conditions, prendre une mesure plus systématique des différents agencements de parenthèses dans des articles de sciences sociales? Un corpus constitué pour l'occasion à partir de deux numéros de Sociologie du travail et d'un numéro du British Journal of Sociology, permet - malgré son caractère numériquement limité (16 articles) - de donner un peu de profondeur empirique aux constats précédents. On peut organiser en quelques remarques les enseignements livrés par le tableau 2 qui est construit à partir du dénombrement des différentes catégories de parenthèses.

Le dénombrement relève un total de 751 parenthèses (voir les modalités de prise en compte en légende au tableau 2). L'agencement principal est syntagmatique, surtout « illustratif » $(n=457$, soit : $60,85 \%)$ et nettement moins « incident » ( $n=184$, soit : $24,50 \%)$, alors que les parenthèses paradigmatiques sont ici exclusivement homologiques $(n=58$, soit : 7, 72\%). L'agencement illustratif des parenthèses syntagmatiques est assez homogène dans l'ensemble du Corpus : elles servent surtout à rapporter des éléments empiriques, comme témoignages du propos tenu dans la partie antérieure de la phrase; et, dans une moindre mesure à fournir des éléments de définition. Cette investigation permet aussi de voir la concurrence qui s'exerce de facto entre la parenthèse et la note de bas de page : les parenthèses incidentes de certains articles sont potentiellement des notes de

sont de moi. On voit qu'ici « paradigme » correspond à ce que Saussure appelle « syntagme ». Perpendiculairement à ce premier axe, «la thématisation prend pour départ l'enchaînement saisi cette fois dans son vol, trajectoire qui se mue en sens. La pensée ne va plus vers le thème créé mais part de la façon de créer pour en donner le principe par une abstraction de même nature que l'autre, mais dirigée transversalement » (op. cit., p. 30) — c'est Cavaillès qui souligne. Cette distinction a l'avantage de souligner que le paradigme (le syntagmatique saussurien) n'est pas seulement singularité d'un enchaînement qui qualifie d'une certaine manière (e.g. en baisse) un objet (e.g. l'usage de la contraception chez les mineures en France) : il définit une opération transposable en chacun de ses points (e.g. en hausse; chez telle catégorie de mineures; dans les pays voisins), qu'il revient à la thématisation non seulement de proposer, mais surtout d'effectuer avec rigueur. La perspective de devoir affronter les effets de la coexistence des sens de paradigme chez Saussure, Kuhn et Cavaillès m'a poussé à un repli sur Saussure.

26. Je choisis d'écrire «expérientiel» ce qui renvoie à une expérience accumulée. J'écrirais « expérienciel » pour une expérience en train de s’opérer (de s'expériencier).

27. On examinera à la fin de cette section un cas assez intéressant d'hybridation : des parenthèses syntagmatiques quasi-homologiques (correspondant en gros à ce que S. Boucheron appelle «parenthèses d'alternative »). 
bas de page, mais n'ont pas bénéficié de ce traitement, le plus souvent par souci d'allègement rhétorique. L'usage des incidentes correspond presque toujours à la désignation d'un prolongement de l'analyse sur certains aspects induits, voire secondaires. Cette pratique ne singularise pas vraiment l'espace anglophone, mais le fait qu'il y soit plus souvent mis en ouvre permet de bien voir son rôle dans la dynamique de l'écriture (un intermédiaire, entre le texte et la note de bas de page, peut-être en rapport avec le fait que les notes anglophones sont très souvent en fin de texte).

Si l'on se reporte au tableau 2, il apparaît assez nettement : 1/que dans les articles publiés en français, le recours aux parenthèses est plus courant (en moyenne 52,50 contre 41,75 pour les anglophones $\left.{ }^{28}\right) ; 2$ /qu'elle sont plus illustratives $(n=289$; $68,81 \%)$ que dans les articles publiés en anglais $(n=168 ; 50,76 \%) ; 3 / q u e$ les articles publiés en anglais ont plus volontiers recours aux parenthèses incidentes $(n=110$; $33,23 \%)$ et homologiques $(n=41 ; 12,38 \%)$ que les articles publiés en français (respectivement : $\mathrm{n}=74 ; 17,62 \%$ et $\mathrm{n}=17 ; 4,04 \%$ ).

Pour autant, on ne peut exclure qu'il y ait, ici comme là-bas, une forme de contrôle indirect de l'usage des parenthèses : à la "constriction syntaxique " (Julien Gracq, cité par S. Boucheron, op. cit., p.11) par laquelle la Langue tend à contrôler le Discours, s'ajoute peut-être une forme de constriction politique. En d'autres termes : une forme de droit d'entrée dans le métier, qui se paierait d'une réduction du nombre de parenthèses. En l'absence de moyens d'établir de manière simple un corpus de données anglophones sur ce point, on peut au moins le vérifier sur l'espace francophone. Les lauréats du « Prix du Jeune Auteur» de la revue Sociologie du travail, en 2006, 2008 et 2009 (soit 9 auteurs) ont au total utilisé 359 parenthèses, ce qui représente une moyenne de 39,9 parenthèses par article (tous types confondus) ${ }^{29}$. Pour la comparaison, puisons dans le corpus examiné ci-dessus des articles d'auteurs publiant dans cette revue (soit 5 articles) en vérifiant empiriquement qu'il ne s'agit pas de débutants. Nous aboutissons à 352 parenthèses, soit une moyenne de 70,4 par article. Une observation attentive

28. On ne peut évidemment pas écarter complètement une explication purement linguistique de cette différence, qui renverrait à des homéostasies stylistiques différentes de l'anglais et du français.

29. Prix du jeune auteur 2006 (Sociologie du travail, 49-3, 2007) : Pilmis O., « Des "employeurs multiples" au "noyau dur d'employeurs" : relations de travail et concurrence sur le marché des comédiens ", pp. 297-315 (n=39); Rubbers B., « Retour sur le "secteur informel”. L'économie du Katanga face à la falsification de la loi », pp. 316-329 (n=32); Giraudeau M., "Le travail entrepreneurial ou l'entrepreneur schumpeterien performé », pp. 330-350 ( $n=41)$. Prix du jeune auteur 2008 (Sociologie du travail, 51-3, 2009) : Gayon V., "Un atelier d'écriture internationale : l'OCDE au travail. Eléments de sociologie de la forme "rapport" », pp. 324-342 $(\mathrm{n}=90)$; Fabros A.S.L., " Global Economy of Signs and Selves : a View of Work Regimes in Call Centers in the Philippines », pp. 343-360 (n=14); Bertrand J., "Entre "passion" et incertitude : la socialisation au métier de footballeur ", pp. 361-378 $(n=75)$. Pour les lauréats de 2009, la liste figure à la suite du tableau 2 ([6,7 et 8$]$. Les établis y sont notés [ 1 à 5$]$. NB : la revue ne semble pas avoir publié de lauréats pour 2007. 
montre que l'article qui utilise le plus de parenthèses est aussi celui qui se sert le plus de la palette des possibles en la matière : en particulier, le recours à des parenthèses homologiques y est plus fréquent. Certes, les autres auteurs de l'échantillon (appelons-les : «établis ») n’ont pas nécessairement une position académique nettement supérieure aux lauréats du Prix et il faudrait donc se garder de toute extrapolation à partir de cette première simulation d'analyse, en raison de l'incertitude sur les éléments d'interprétation susceptibles d'éclairer cette situation. Néanmoins, on peut se demander si ce n'est pas là l'effet conjugué d'un autocontrôle et d'une incitation : l'entrée dans l'espace de la publication se paie-telle d'une forme d'autocensure portée par une idéologie de la sobriété; les auteurs primés, dont les textes sont en principe publiés "tels quels", sont-ils néanmoins invités explicitement à une relecture visant à alléger le style en supprimant de «trop nombreuses parenthèses »?

\subsection{Les nouages syntagmatiques de l'homologique et de l'heuristique}

L'écriture de Bourdieu permet de réaliser une première observation plus en profondeur de l'agencement métalinguistique qu'autorisent les parenthèses. Pour en rendre compte, il faut avoir à l'esprit deux constantes de son style. Premièrement, sur un plan intra-théorique, il s'agit d'une écriture qui établit en permanence une correspondance entre le terrain qu'elle explore (un champ, dans son vocabulaire - par exemple le champ littéraire dans lequel s'inscrira l'œuvre de Flaubert) et d'autres espaces dont ce terrain traduit les enjeux d'une manière spécifique (par exemple l'espace des positions sociales, qui n'explique pas directement Flaubert, mais sans la prise en compte duquel on ne peut le comprendre sociologiquement). Le concept bourdieusien qui rend compte de ce geste est l'homologie. Les parenthèses, plus encore que les notes, ont pour fonction de le rappeler d'une manière en quelque sorte sténographique. Deuxièmement, sur un plan plus stylistique (au sens d'affirmation de singularités), il s'agit d'une écriture qui définit toujours un programme de recherche en même temps qu'elle expose des résultats de recherche. Certaines parenthèses sont alors des invitations à l'analogie. Si l'on considère que tout outil donnant la clé transposable d'un énoncé est un métalangage, toute parenthèse paradigmatique doit être considérée comme un marqueur de métalangage dense. Dans l'exemple étudié ci-après ${ }^{30}$, l'homologie est celle du champ religieux et du champ économique. Je fais figurer en gras l'espace paradigmatique homologique pour le distinguer de l'espace paradigmatique heuristique (ici en caractères italiques), bien que, chez Bourdieu, tous deux soient des expressions de l'homologie des champs, l'une strictement analogique, l'autre expansive :

30. On se réfère ici à : Bourdieu P., " Genèse et structure du champ religieux », Revue Française de Sociologie, 12-3, 1971, p. 295-334. 
[...] la contestation prophétique (ou hérétique) del'Église menacel'existence même de l'institution ecclésiastique lorsqu'elle met en question non seulement l'aptitude du corps sacerdotal à remplir sa fonction proclamée $(<1>a u$ nom $d u$ refus de la "grâce institutionnelle») mais aussi la raison d'être du sacerdoce $(<2>$ au nom $d u$ principe $d u$ «sacerdoce universel $»)$ et [...] elle ne peut s'achever que par la suppression du prophète (ou de la secte), par la violence physique ou symbolique (excommunication) //parenthèse syntagmatique illustrative// [...]

La phrase continue ainsi :

[...] à moins que la soumission du prophète (ou du réformateur), i.e. la reconnaissance de la légitimité du monopole ecclésiastique (<3>et de la hiérarchie qui le garantit), n'autorise l'annexion par la canonisation (e.g. Saint-François d'Assise) //parenthèse syntagmatique illustrative//(p. 322).

Commentons brièvement au moins les parenthèses heuristiques, notées $<1>$, $<2>,<3>$. Elles introduisent le principe explicatif là où le déroulement syntagmatique dirait simplement le fait : $<1>$ la fonction sacerdotale doit reposer sur une transcendance pour être légitime; $<2>$ elle ne saurait être un monopole sans être contraire à l'égale compétence de la communauté des fidèles; $<3>$ elle ne doit son monopole qu'au primat du domestique (la hiérarchie) sur le civique (l'égalité). Mais comme le disent les trois parenthèses homologiques, cette contestation (hérétique) inspirée tangue entre l'installation d'une dissidence collective (la secte) et l'affirmation d'un programme industriel (le réformateur) tous deux susceptibles de réintroduire du domestique ${ }^{31}$. Si toutes les parenthèses de cet extrait participent métalinguistiquement à l'établissement d'une épaisseur intra- et méta-théorique, elles n'ont pas la même densité : les syntagmatiques (excommunication et canonisation) sont comme des faits qui auraient besoin des paradigmatiques homologiques et heuristiques pour que l'on comprenne leur parfaite symétrie, là où le lecteur pressé ne verrait qu'une opposition radicale.

Soit un exemple, sur une seule phrase, de la variété des ressources utilisées (identifiées ici seulement entre barres obliques) :

[...] et, plus précisément, imposer les observances rituelles qui, vécues comme la condition de la sauvegarde de l'ordre cosmique et de la subsistance du groupe (le cataclysme naturel jouant dans certains contextes le rôle que la révolution politique joue en d'autres) //parenthèse paradigmatique heuristique//, tendent en fait (une des fonctions principales du rite étant de rendre possible la réunion de principes mytho-logiquement séparés, comme le masculin et le féminin [...] etc.) //parenthèse syntagmatique incidente// à perpétuer les relations fondamentales de l'ordre social, c'est transmuer la transgression [...] en sacrilège enfermant sa propre sanction, quand ce n'est pas rendre impensable l'idée même de la transgression de frontières si parfaitement "naturalisées " (parce qu'intériorisées comme principes de structuration du monde) //parenthèse syntagmatique incidente// qu'elles ne peuvent être abolies qu'au prix

31. Le lexique majeur utilisé ici est celui de Boltanski et Thévenot (1991). 
d'une révolution symbolique (e.g. la révolution copernicienne et galiléenne d'un côté, machiavélienne de l'autre) // parenthèse syntagmatique illustrative //[...] (p. 330).

À ce premier stade de l'examen, il en va des parenthèses comme des notes : chaque type peut contenir des inscriptions métalinguistiques, mais a priori le lieu privilégié de leur densité serait la parenthèse paradigmatique. La parenthèse syntagmatique ${ }^{32}$, souvent énumérative (fonctionnant sur le mode « etc. ${ }^{33}$ ), peut être un lieu de métalangage lorsqu'elle se veut résumante ${ }^{34} \mathrm{ou}$, mieux encore critique. Ainsi, dans un texte qui comporte - notons-le sans pouvoir le commenter - un nombre non négligeable de guillemets critiques polémiques, l'auteur écrit :

Si le réalisme ne paraît pas, en effet, très influencé par l'information sur les risques (contrairement à ce que suggèrent le risk assessment et, dans le débat étudié ici, le débat entre W. Viscusi et D. Nelkin), parce qu'il est lui-même fonction de la construction sociale de la perception, cela ne veut pas dire qu'il soit toujours biaisé par des positions catégorielles (comme le soutiennent M. Douglas et d'autres auteurs culturalistes) (p. 40).

"Contrairement» et "comme le soutiennent» aident à comprendre la dimension métalinguistique parce que métathéorique des deux parenthèses. Mais on peut aller plus loin, par exemple à l'occasion d'une controverse. Rencontrée cidessus à propos de la pratique des notes, la controverse Chamboredon - Boudon en offre aussi une illustration. Chamboredon pratique la parenthèse comme un mode d'écriture baroque, c'est-à-dire que chaque parenthèse est un tiroir où le lecteur peut / doit se perdre. La réponse de J.-C. Chamboredon ${ }^{35}$ contient 29 paires de parenthèses dont la grande majorité consiste en allusions lettrées et ironiques visant, comme il se doit dans une polémique, à rectifier des énoncés tronqués par excès (mauvaise interprétation) ou par défaut (silence coupable).

Si Bourdieu illustre au plus haut point la pratique de la parenthèse homologique, certains auteurs anglophones ont recours de manière régulière à une variante syntagmatique qui joue sur l'écart lexical et introduit ainsi une forme d'homologie (parenthèse quasi-homologique). Par exemple, Nick Crossley - utilisant, comme

32. Les exemples qui suivent sont tous pris dans: Duclos D., «La construction sociale du risque : le cas des ouvriers de la chimie face aux dangers industriels ", Revue Française de Sociologie, 28-1, 1991 p. 17-42.

33. Soit un usage énumératif reposant sur une illustration expérientielle : «[...] comment les producteurs envisageaient les rapports entre les avantages de l'activité industrielle (emploi, demande de produits, rendement de l'investissement, etc.) et ses incidences néfastes éventuelles $[\ldots] »($ p. 18).

34. «La thèse selon laquelle c'est le contexte qui crée le sentiment du risque (thèse que nous avons plutôt appuyée ici tout en la nuançant) pourrait passer pour contradictoire avec l'existence de cette réserve de perceptions individuelles du risque "vrai" » (p. 37). On voit ici que la parenthèse syntagmatique résumante a une dimension métalinguistique.

35. Chamboredon, J.-C., "Réponse à MM. Boudon et Bourricaud, auteurs du dictionnaire », Revue française de sociologie, XXV-2, 1984, pp. 332-333. 
beaucoup de chercheurs et de caricaturistes, ce que Sabine Boucheron appelle une "parenthèse d'alternative » (Boucheron, 1997) — joue sur les mots pour construire un titre avec parenthèse quasi-homologique : le titre (Net)Working out : social capital in a private health $c l u b^{36}$ indique, à la fois, qu'il y a construction de capital social et que cette construction se fait via le réseau. Dire Networking serait limiter cette élaboration (working out) à sa dimension réticulaire, ce qui pourrait écraser les nuances de ce tissage observé par l'auteur lui-même (tout n'est pas réseau). Bien entendu, on trouve aussi dans l'espace francophone des pratiques de ce genre.

On peut montrer l'articulation de ces deux agencements dans deux textes du corpus. Benny Geys et Zuzana Murdoch, dans un texte comportant 84 parenthèses et un taux élevé de parenthèses homologiques $(n=18 ; 21,4 \%)$, ont recours à de nombreux cas d'homologies à la manière de Bourdieu : 1 /soit en se situant à un niveau métathéorique (comme: We are now in position to consider wether or not both interpretations of [or methodological approach to] bridging versus bonding social networks lead to analogous results ${ }^{37}$ ); 2 /soit, plus fréquemment, à un niveau intra-théorique, ce qui est favorisé par l'objet même de l'article (une étude sur l'imprécision de la distinction entre bridging et bonding). Chaque fois qu'il est question de pratiques tournées vers le pontage (bridging) entre deux réseaux, elle est mise en correspondance avec les pratiques de collage (bonding) internes à un réseau. D’où la commodité des parenthèses homologiques à la manière de Bourdieu. Par exemple - riche de deux homologies : Associations at the lower (higher) end of the scale are therefore seen as predominently bridging (bonding) [... $]^{38}$

Le texte de Nick Crossley (op. cit.), à peu près deux fois moins utilisateur de parenthèses $(n=49)$, a recours à la quasi-homologie par trois fois (en comptant le titre, examiné ci-dessus). Mais trois autres agencements méritent l'examen. Ayant en quelque sorte d'emblée (par le titrage homologique) posé l'horizon de son analyse, Crossley utilise trois parenthèses syntagmatiques qui ne sont réductibles à aucun des usages mentionnés ci-dessus : elles ne sont compréhensibles que parce qu'elles explicitent un sous-entendu et par là en définissent la portée générale, abstraite. C'est précisément cela que visait, dans un autre contexte, la notion de paradigme chez Cavaillès (rappelons-le, c'est le syntagme saussurien). La portée métalinguistique de ces parenthèses réside dans cette explicitation. Explicitation d'une quantité : «[...] what they might conceptualize as their (collective) time [...]"; ou encore : «[...] whereby it was (collectively) framed [...]». Explicitation d'une nécessité : «[...] can obstruct (necessary) forms of state intervention [...] ». Explicitation d'un degré : «[...] a further type of (moderate) conflict [...]»; ou encore :

36. Crossley N., « (Net)Working out : social capital in a private health club », The British Journal of Sociology, 59-3, 2008, p. 475.

37. Geys B., Murdoch Z., " How to make head or tail of "bridging" and "bonding"? : addressing the methodological ambiguity», The British Journal of Sociology, 59-3, 2008, p. 441.

38. Op. cit., p. 439. 
"[...] in a (mildly) conflictual manner [...] ${ }^{39}$. Parenthèses paradigmatiques homologiques et parenthèses syntagmatiques apparaissent ainsi nouées, parce que l'homologie n'y repose pas sur l'identité de deux pratiques, mais sur leur comparabilité, sur l'existence d'une échelle de mesure des phénomènes.

\section{La coexistence pacifique des agencements métalinguistiques}

Nous avons, au cours de cet examen, rencontré de nombreux agencements métalinguistiques dans les travaux publiés des sociologues, aucun d'entre eux n'ayant le caractère d'un langage formalisé, aucun d'entre eux n'ayant non plus de caractère systématique. Pour autant, cette situation ne déroge pas aux effets des choix théoriques annoncés au début : même formulés en langage naturel, même discontinus, les agencements métalinguistiques sont ontologiques au même titre que ceux qui déploient des résultats d'enquête ou explicitent des méthodologies. Ontologiques, ils spécifient des êtres (des théories, des concepts, des méthodes, des terrains), leurs modes possibles d'engagement dans l'argumentation, leurs interrelations, mais ils sont assemblés sous une contrainte de connivence : comme le locuteur ordinaire est habilité et appelé à repérer ce qu'il y a d'éventuellement métalinguistique dans un énoncé, le lecteur putatif de sciences sociales (celui dont l'auteur présuppose l'intérêt et dont la revue présuppose l'existence) est plus ou moins habilité et appelé à relever les traces à peine visibles de chemins et de cheminements conceptuels qui relèveraient de catégories alors qu'ils ne sont le plus souvent mentionnés que dans leur singularité, jusqu'à cette limite que serait un écart lexical isolé, pourvu d'une définition localiste. Cet effet de connivence, qu'il faut bien qualifier de "lettré », paraît affecter plus fortement les énoncés métalinguistiques que les autres et autorise bien évidemment malentendus et passages en force. Cet effet n'est sans doute levé qu'en situation d'épreuve, lorsque la montée en généralité due à la présence sur l'autoroute argumentative d'un obstacle impossible à ignorer oblige l'énonciateur à assembler les différents éléments métalinguistiques en référence explicite à un principe supérieur de classement (selon le modèle fourni par : Boltanski \& Thévenot, 1991). Je n'ai pas opté ici pour une présentation détaillée de ces mœurs, faute d'avoir pu collecter des matériaux suffisamment variés pour étayer ce qui reste de ce fait une hypothèse régulatrice.

Dès lors qu'on se restreint à une approche topologique (ici : les notes et les parenthèses ont été considérées comme des lieux d’hébergement propices), certaines des relations ontologiques entre les différents éléments agencés se dégagent. On observe dans les deux lieux une sorte de découplage entre métathéorie et métalangage : si les agencements qui privilégient des considérations métathéoriques sont des résultats d'assemblages métalinguistiques, nombreux sont les dispositifs métalinguistiques qui ont une fonction exclusivement intrathéorique.

39. Op. cit., pp. $481,485,490,494,497$. 
On est alors fondé à se demander s'il ne faudrait pas différencier, du moins pour les sciences sociales, des métalangages génériques et des métalangages spécifiques. Pourtant, ce pourrait bien être une fausse bonne idée. Les sciences sociales, même si elles sont loin d'avoir achevé leur croissance et d'avoir trouvé leur régime de croisière ont, depuis de nombreuses années, stabilisé des régimes d'énonciation engageant des agencements métalinguistiques intra- et méta-théoriques auxquels elles accordent des légitimités scientifiques équivalentes. Il faut donc prendre au sérieux la conception de l'ontologie, comme politique pragmatique, énoncée dans notre première partie : lorsqu'un agencement métalinguistique est mis en œuvre (enacted) dans un texte de sciences sociales, il assemble un système de relations entre les actants qu'il engage (une ontologie), quels que soient le degré et l'ampleur de la thématisation métathéorique à laquelle il recourt. Et, à voir la coexistence plutôt pacifique, dans un même espace de publication, des articles qui majorent ou minorent la thématisation, qui explicitent ou implicitent leur épistémologie, il semble bien que l'on doive se satisfaire de cet état pluriel des ontologies ${ }^{40}$. C'est pourquoi, fidèle à une ontologie des "passages », on a esquissé ici des explication en continuum, depuis le leurre métalinguistique jusqu'aux métalangages denses.

Les écrits sociologiques ne traduisent pas un stylos préétabli ou implicite (une ontologie générique), ils le configurent à chaque énonciation. Mais en tant que doxa d'une discipline qui est supposée engager la référence à un terrain, le stylos sociologique le plus couramment observable met à distance respectable les agencements métathéoriques envahissants : il les évacue des thèses (et les réserve plus ou moins pour les travaux des chercheurs aguerris); il en confine certains en des lieux textuels privilégiés (ici : notes et parenthèses) et en dissémine d'autres sur l'ensemble du texte. On trouvera certains stilus pour assembler autrement les agencements (plus continûment ou plus manifestement), mais il faut bien admettre que cette ontologie n'a pas une force illocutoire plus grande. En outre, il semble difficile, en l'état actuel d'une épistémologie de l'écriture des sciences sociales, de prendre vraiment la mesure du continuum des ontologies en vigueur à un moment donné. C'est cette topologie énonciative que l’on a voulu éclairer ici, sans lui donner la force d'une topique, en mettant l'accent sur les passages d'un état à un autre.

\section{Références bibliographiques}

Anscombre, Jean-Claude (1995), "La théorie des topoï : sémantique ou rhétorique? », Hermès 15 (Argumentation et rhétorique I), CNRS Éditions, pp. 185-198.

40. Toutes proportions gardées, cette coexistence est l'effet, dans l'accessibilité au discours scientifique, des formes de réflexivité (cryptique, hermétique et opaque) que Fontanille jugeait aussi «intéressantes et productives » que la réflexivité ostensive, en termes de modélisation (Fontanille, 2003, p.10 de la version fournie par l'auteur sur son site). 
Auroux, Sylvain (1979), «Catégories de métalangages », Histoire Épistémologie Langage, Tome 1, fascicule 1, pp. 3-14.

Authier-Revuz, Jacqueline (2002), «Du Dire “en plus” : dédoublement réflexif et ajout sur la chaîne", in J. Authier-Revuz \& M.C. Lala (dir.), Figures d'ajout. Phrase, texte, écriture, Paris, Presses de la Sorbonne Nouvelle, pp. 147-167.

BeCKer, Howard S. (1988), Les mondes de l'art, Paris, Flammarion.

Boltanski, Luc \& Thevenot, Laurent (1991), De la justification. Les économies de la grandeur, Paris, Gallimard.

Boucheron Sabine (1997), «De la légèreté des parenthèses : morphèmes, logatomes et autres graphèmes », L'information grammaticale, 73, pp. 10-11.

http://www.persee.fr/web/revues/home/prescript/article/igram_0222-9838_1997_ num_73_1_3556

-, (1999), «La ponctualité dans le texte : parenthèses, sujets et linéarité dans l'incipit du Palace de Claude Simon », Recherches linguistiques de Vincennes, 28, pp. 33-40.

Cavailles, Jean (1960), Sur la logique et la théorie de la science, Paris, P.U.F., $2^{2}$ édition ( ${ }^{\text {re }}$ édition : 1947).

Dupas, Jean-Jacques (2008), « La notation polonaise », Tangente, 120, pp.30-31.

Fontanille, Jacques (2003), "Introduction : Modélisation et textualité», Modèles linguistiques, $24 / 1$.

Fuchs, Catherine (1995), Paraphrase et énonciation, Paris, Ophrys.

GAYon Jean (1998), «De l'usage de la notion de style en histoire des sciences », in GAYon J., Gens J.-C. (dir.), La rhétorique: Enjeu de ses résurgences, Bruxelles, Ousia, pp. 162-181.

Genette, Gérard (1987), Seuils, Paris, Ed. du Seuil.

Grafton, Anthony (1998), Les origines tragiques de l'érudition. Une histoire de la note en bas de page, traduction de P.A. Fabre, Paris, Le Seuil.

Greimas, Algirdas J. \& Courtes, Joseph (1979), Sémiotique. Dictionnaire raisonné de la théorie du langage I, Paris, Hachette.

-, (éds, 1986), Sémiotique. Dictionnaire raisonné de la théorie du langage II, Paris, Hachette.

Hadria, Armand \& Gaussot, Pierre (2007), «Du bon usage des parenthèses », Défense de la langue française, 225, pp. 36-38.

Hardie, Iain, McKenzie, David (2007), « Assembling an Economic Actor: The Agencement of a Hedge Fund », The Sociological Review, vol. 55, 1, pp. 57-80.

Kunn, Thomas S. (1970), "Reflections on my Critics », in Lakatos, Imre \& Musgrave Allan, Criticism and the Growth of Knowledge, Cambridge, Cambrige University Press.

Lefebvre, Julie (2004), «Représentation du "discours autre" et note de bas de page : un jeu sur deux lignes », in Juan-Manuel Lopez-Muñoz, Sophie Marnette \& Laurence Rosier (éds), Le Discours rapporté dans tous ses états, Paris, L'Harmattan, pp. 428-436. 
MoL, Annemarie (1999), " Ontological Politics. A Word and some Questions », in J. LAW \& J. Hassard, Actor-Network Theory and After, Oxford, Blackwell (The Sociological Review Monographs), pp. 74-89.

Moser, Ingunn \& LaW, John, « Good passages. Bad passages ", in J. Law \& J. Hassard, Actor-Network Theory and After, Oxford, Blackwell (The Sociological Review Monographs), pp. 196-219.

ReY, Alain (1979), La terminologie : mots et notions, Paris, PUF.

Rey-Debove, Josette (1978), Le métalangage. Etude linguistique du discours sur le langage, Paris, Le Robert.

Stockinger, Peter, Introduction to Digital Audiovisual Archives, London-New York, John Wiley and sons.

Trépos, Jean-Yves (2011), L'écriture de la sociologie. Essai d'une épistémologie du style en sciences sociales, Paris, L'Harmattan.

VAndendorpe, Christian (1990), «Paradigme et syntagme. De quelques idées vertes qui ont dormi furieusement ", Revue québécoise de linguistique théorique et appliquée vol. 9, nº 3, pp. 169-193.

\section{Annexes}

Tableau 1 : Pratiques de la note infrapaginale dans quatre revues de sciences sociales (2000-2001)

\begin{tabular}{|c|c|c|c|c|}
\hline & $\begin{array}{c}\text { Revue } \\
\text { française de } \\
\text { sociologie } \\
\left(224 \mathrm{p} / \mathrm{n}^{\circ}\right)\end{array}$ & $\begin{array}{c}\text { Sociologie du } \\
\text { travail } \\
\left(156 \mathrm{p} / \mathrm{n}^{\circ}\right)\end{array}$ & $\begin{array}{c}\text { The } \\
\text { Sociological } \\
\text { Review } \\
\left(156 \mathrm{p} / \mathrm{n}^{\circ}\right)\end{array}$ & $\begin{array}{l}\text { Science as } \\
\text { Culture } \\
\left(136 \mathrm{p} / \mathrm{n}^{\circ}\right)\end{array}$ \\
\hline \multicolumn{5}{|l|}{ Nombre de références } \\
\hline $2000-4$ & 165 [6] & 17 [6] & 69 [7] & 06 [6] \\
\hline $2001-1$ & $131[6]$ & $95[8]$ & $63[6]$ & $31[6]$ \\
\hline $2001-2$ & $130[6]$ & $96[7]$ & $41[8]$ & $37[7]$ \\
\hline Total & $426[18]$ & $208[21]$ & $173[21]$ & $74[19]$ \\
\hline \multicolumn{5}{|l|}{ N. de signes } \\
\hline 2000-4 & 41040 & 50000 & 24100 & 3825 \\
\hline 2001-1 & 49230 & 25700 & 22300 & 9945 \\
\hline $2001-2$ & 45090 & 5700 & 13800 & 17765 \\
\hline Total & $\begin{aligned} 135360 \\
\mathrm{r} 1=201,4 \\
\mathrm{r} 2=317,7\end{aligned}$ & $\begin{array}{l}36400 \\
\mathrm{r} 1=77,7 \\
\mathrm{r} 2=175,0\end{array}$ & $\begin{array}{l}60800 \\
\mathrm{r} 1=129,9 \\
\mathrm{r} 2=351,4\end{array}$ & $\begin{array}{l}31535 \\
\mathrm{r} 1=77,2 \\
\mathrm{r} 2=426,1\end{array}$ \\
\hline
\end{tabular}

NB : on n'a pas intégré les éventuelles notes des «comptes rendus » et les notes dévolues à des remerciements dans le décompte. Entre crochets, le nombre d'articles par numéro.

$\mathrm{r} 1=\mathrm{n}$. de signes / n. de p. des $3 \mathrm{n}^{\circ}$

$\mathrm{r} 2=\mathrm{n}$. de signes $/ \mathrm{n}$. de notes 
Tableau 2 : L'usage des parenthèses dans 16 articles de sociologie

\begin{tabular}{|c|c|c|c|c|c|c|}
\hline \multirow[b]{2}{*}{ Parenthèses } & \multicolumn{3}{|c|}{ SYNTAGMATIQUES } & \multicolumn{2}{|c|}{ PARADIGMATIQUES } & \multirow[t]{2}{*}{$\mathrm{T}$} \\
\hline & 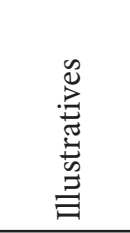 & 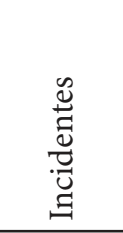 & 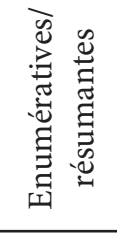 & 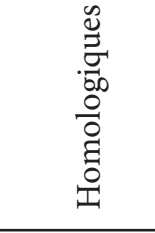 & 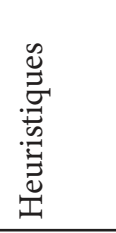 & \\
\hline Buscatto \& Marry & 11 & 4 & 6 & 6 & 0 & 27 \\
\hline Schweitzer & 19 & 0 & 0 & 0 & 0 & 19 \\
\hline de Gasquet & 29 & 23 & 4 & 4 & 0 & 60 \\
\hline Backouche et al. & 77 & 19 & 10 & 1 & 0 & 107 \\
\hline Guillaume \& Pochic & 95 & 27 & 13 & 4 & 0 & 139 \\
\hline Benquet & 19 & 0 & 0 & 0 & 0 & 19 \\
\hline Pollard & 24 & 1 & 3 & 0 & 0 & 28 \\
\hline Plessz & 15 & 0 & 4 & 2 & 0 & 21 \\
\hline sous-total F & $\begin{array}{c}289 \\
68,81 \% \\
\end{array}$ & $\begin{array}{c}74 \\
17,62 \% \\
\end{array}$ & $\begin{array}{c}40 \\
9,53 \% \\
\end{array}$ & $\begin{array}{c}17 \\
4,04 \% \\
\end{array}$ & 0 & $\begin{array}{c}420 \\
100 \% \\
\end{array}$ \\
\hline Acker & 4 & 2 & 0 & 0 & 0 & 6 \\
\hline Bielby & 4 & 4 & 2 & 0 & 0 & 10 \\
\hline Li et al. & 43 & 12 & 2 & 3 & 0 & 60 \\
\hline Paterson & 36 & 27 & 0 & 4 & 0 & 67 \\
\hline Geys \& Murdoch & 43 & 20 & 3 & 18 & 0 & 84 \\
\hline Elder-Vass & 5 & 13 & 0 & 7 & 0 & 25 \\
\hline Crossley & 23 & 17 & 3 & 6 & 0 & 49 \\
\hline Jenks \& Smith & 10 & 15 & 2 & 3 & 0 & 30 \\
\hline sous-total GB & $\begin{array}{c}168 \\
50,76 \% \\
\end{array}$ & $\begin{array}{c}110 \\
33,23 \% \\
\end{array}$ & $\begin{array}{c}12 \\
3,63 \% \\
\end{array}$ & $\begin{array}{c}41 \\
12,38 \% \\
\end{array}$ & 0 & $\begin{array}{c}331 \\
100 \% \\
\end{array}$ \\
\hline Total & $\begin{array}{c}457 \\
60,86 \% \\
\end{array}$ & $\begin{array}{c}184 \\
24,50 \% \\
\end{array}$ & $\begin{array}{c}52 \\
6,92 \% \\
\end{array}$ & $\begin{array}{c}58 \\
7,72 \% \\
\end{array}$ & 0 & $\begin{array}{c}751 \\
100 \% \\
\end{array}$ \\
\hline
\end{tabular}

\section{Lecture :}

L'article de M. Buscatto et C. Marry comporte 27 parenthèses, dont 11 sont illustratives, 4 incidentes, 6 énumératives/résumantes et 6 homologiques. L'ensemble des articles publiés en français (sous-total F) compte 420 parenthèses, dont : 289 (68,81\%) sont illustratives, $74(17,62 \%)$ sont incidentes, 40 (9,53\%) sont énumératives/résumantes et 17 (4,04\%) sont homologiques.

NB : Les parenthèses servant à énumérer des références n’ont pas été dénombrées, mais celles qui renvoyaient à des figures ou tableaux et celles qui explicitaient des sigles l'ont été (parenthèses illustratives).

$\left(\chi^{2}\right.$ significatif à p.01) 
Liste des articles utilisés :

[1] Buscatto M., Marry C., « Le "plafond de verre dans tous ses éclats”. La féminisation des professions supérieures au xx ${ }^{\mathrm{e}}$ siècle », Sociologie du travail, 51, 2, 2009, pp. 170-182.

[2] Schweitzer S., «Du vent dans le ciel de plomb? L'accès des femmes aux professions supérieures, $\mathrm{XIX}^{\mathrm{e}}-\mathrm{XX}^{\mathrm{e}}$ siècles », Sociologie du travail, 51, 2, 2009, pp. 183-198.

[3] DE Gasquet B., «La barrière et le plafond de vitrail. Analyser les carrières féminines dans les organisations religieuses ", Sociologie du travail, 51, 2, 2009, pp. 218-236.

[4] Backouche I., Godechot O., Naudier D., «Un plafond à caissons : les femmes à l'EHESS », Sociologie du travail, 51, 2, 2009, pp. 253-274.

[5] Pochic S., Guillaume C., "Les carrières de cadres au cœur des restructurations : la recomposition des effets de genre? L'internationalisation d'un groupe français en Angleterre et en Hongrie », Sociologie du travail, 51, 2, 2009, pp. 275-299.

[6] Benquet M., «Les raisons de l'action collective : retour sur la mobilisation improbable des salariés d'hypermarchés », Sociologie du travail, 52, 3, 2010, pp. 305-322.

[7] Pollard J., "Soutenir le marché : les nouveaux instruments de la politique du logement », Sociologie du travail, 52, 3, 2010, pp. 323-339.

[8] Plessz M., «Les ouvriers en Europe centrale : la dissolution d'une catégorie sociale dans les statistiques ", Sociologie du travail, 52, 3, 2010, pp. 340-358. 